\title{
Generalized bloch equations for optical interactions in confined geometries
}

\author{
Christian Girard ${ }^{\mathrm{a}, *}$, Olivier J.F. Martin ${ }^{\mathrm{b}}$, Gaëtan Lévèque ${ }^{\mathrm{b}}$, \\ Gérard Colas des Francs ${ }^{\mathrm{c}}$, Alain Dereux ${ }^{\mathrm{c}}$ \\ a Centre dElaboration des Matériaux et dEtudes Structurales (CNRS), 29 Rue Jeanne Marvig, F-31055 Toulouse, France \\ ${ }^{\mathrm{b}}$ Nanophotonics and Metrology Laboratory, Swiss Federal Institute of Technology Lausanne (EPFL) CH-1015 Lausanne, Switzerland \\ ${ }^{\mathrm{c}}$ Equipe doptique Submicronique, Laboratorie de Physique de l'Université de Bourgogne 9 Av. A. Savary, F-21078 Dijon, France
}

Received 25 August 2004; in final form 29 December 2004

\begin{abstract}
By combining the field-susceptibility technique with the optical Bloch equations, a general formalism is developed for the investigation of molecular photophysical phenomena triggered by nanometer scale optical fields in the presence of complex environments. This formalism illustrate the influence of the illumination regime on the fluorescence signal emitted by a single molecule in a complex environment. In the saturated case, this signal is proportional to the optical local density of states, while it is proportional to the near-field intensity in the non-saturated case.
\end{abstract}

(C) 2005 Elsevier B.V. All rights reserved.

\section{Introduction}

Over the last decade, numerous experimental configurations have been proposed for studying single molecule photophysical phenomena triggered by nanometer size light fields [1-8]. These configurations involve the interaction of microscopic entities with their macroscopic environment. Similar situations, where elements exhibiting very different length scales interact, exist also in solid state physics and materials science [9].

The main objective of this communication is to propose a formalism for analyzing a large class of experimental configurations where various molecular photophysical processes are induced by highly confined optical fields in the presence of a complex environment. The approach is based on the combination of the fieldsusceptibility/Green's-tensor technique [10,11] with the optical Bloch equations [12,13]. The former provides an

\footnotetext{
* Corresponding author. Fax: +33 562257999 .

E-mail address: girard@cemes.fr (C. Girard).
}

accurate electromagnetic description of the system, including the local electric field and local density of states (LDOS) [14]. These parameters are then introduced into the optical Bloch equations to obtain the population evolution of the molecular energy levels and deduce the molecular fluorescence signals [15-17].

Our approach relies on the fact that quantummechanical calculations can be restricted to the molecular system, while the complex optical environment can be treated classically using the Green's tensor. This contrasts markedly with earlier theoretical works on fluorescence in confined geometries where the molecule was treated as a classical emitting dipole [18-21]. Indeed, in the present approach the population equations can be solved in a non-perturbative manner, irrespective of the complexity of the environment seen by the molecules. Like this, one or several molecules including an arbitrary number of energy levels can be investigated. Different experimental illumination regimes can be investigated and effects such as saturation are readily accessible to the technique. These complex effects play 
a central role in the interpretation of single molecule experiments in the presence of highly confined optical fields.

The Letter is organized as follows: A general formalism for the evolution of the different fluorescence signals generated by a molecule placed in a complex environment is first developed. Two limiting cases are then studied in detail to illustrate the dependence of the fluorescence signal on the illumination conditions.

\section{Formalism}

Let us consider a molecular system located at the position $\mathbf{r}_{\mathrm{m}}$ in a complex environment and characterized by its dipole operator $\hat{\boldsymbol{\mu}}$ and Hamiltonian $\mathrm{H}_{0}$ (Fig. 1). The Fourier transform $\mathbf{E}\left(\mathbf{r}_{\mathrm{m}}, \omega\right)$ of the local electric field $\mathbf{E}\left(\mathbf{r}_{\mathrm{m}}, t\right)$ can be obtained everywhere in the system from the generalized field propagator $\mathbf{K}\left(\mathbf{r}, \mathbf{r}^{\prime}, \omega\right)[10]$ :

$\mathbf{E}\left(\mathbf{r}_{\mathrm{m}}, \omega\right)=\int_{v} \mathbf{K}\left(\mathbf{r}_{\mathrm{m}}, \mathbf{r}^{\prime}, \omega\right) \cdot \mathbf{E}_{0}\left(\mathbf{r}^{\prime}, \omega\right) \mathrm{d} \mathbf{r}^{\prime}$,

where $\mathbf{E}_{0}\left(\mathbf{r}^{\prime}, \omega\right)$ represents the Fourier transform of the illumination field $\mathbf{E}_{0}(\mathbf{r}, t)=\mathbf{E}_{0} \cos \left(\omega_{0} t-\mathbf{k}_{0} \cdot \mathbf{r}\right)$, and the integral runs over the entire environment. The dyad $\mathbf{K}\left(\mathbf{r}, \mathbf{r}^{\prime}, \omega\right)$ can be expressed in terms of the optical field susceptibility tensor $\mathbf{S}\left(\mathbf{r}, \mathbf{r}^{\prime}, \omega\right)$ associated with the entire system

$\mathbf{K}\left(\mathbf{r}, \mathbf{r}^{\prime}, \omega\right)=\delta\left(\mathbf{r}-\mathbf{r}^{\prime}\right)+\mathbf{S}\left(\mathbf{r}, \mathbf{r}^{\prime}, \omega\right) \cdot \boldsymbol{\chi}\left(\mathbf{r}^{\prime}, \omega\right)$,

where $\chi$ is the optical susceptibility of the complex environment. Eq. (1) can be solved using a recursive series of Dyson's equations associated with $\mathbf{S}$, leading to the field $\mathbf{E}(\mathbf{r}, \omega)$ in the system [10]. The dipolar coupling Hamilto-

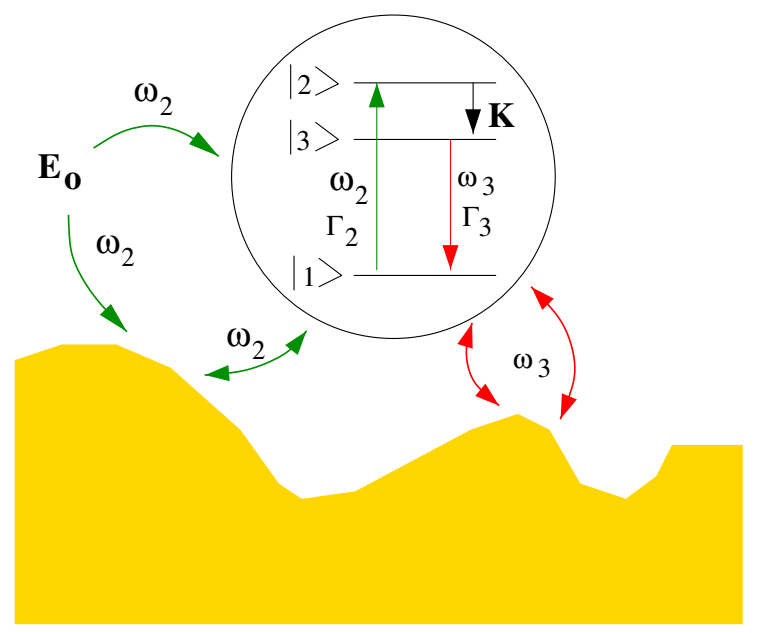

Fig. 1. Schematic representation of a three levels molecular system in interaction with a complex optical environment. Symbols $|1\rangle$ and $|3\rangle$ represent ground single state and first excited singlet, respectively. $K$ characterizes the vibrational relaxation decay of state $|2\rangle$ and $\mathbf{E}_{0}$ is the amplitude of the illumination field. nian $W(t)=-\hat{\boldsymbol{\mu}} \cdot \mathbf{E}\left(\mathbf{r}_{\mathrm{m}}, t\right)$ can then be obtained from the generalized propagator (2). Applying the usual rotating waves approximation that neglects non-resonant terms in the interaction process [12], one obtains

$W(t)=\sum_{\alpha=x, y, z} \frac{\hbar \Omega_{\alpha}\left(\mathbf{r}_{\mathrm{m}}\right)}{2}\left\{\mathrm{e}^{-\mathrm{i}\left(\omega_{0} t+\phi_{\alpha}\left(\mathbf{r}_{\mathrm{m}}\right)\right)}|2\rangle\langle 1|+C . C.\right\}$,

where $\Omega_{\alpha}\left(\mathbf{r}_{\mathrm{m}}\right)$ is the $\alpha$-component of the vectorial Rabi frequency. This quantity is proportional to the $\alpha$-component of the absorption transition dipole $\boldsymbol{\mu}_{2}$

$\Omega_{\alpha}\left(\mathbf{r}_{\mathrm{m}}\right)=-\frac{\mu_{2, \alpha} \mathbf{E}_{\alpha}\left(\mathbf{r}_{\mathrm{m}}\right)}{\hbar}$,

with

$\mathbf{E}_{\alpha}\left(\mathbf{r}_{\mathrm{m}}\right)=\left|\sum_{\beta=x, y, z} \int \mathbf{K}_{\alpha \beta}\left(\mathbf{r}_{\mathrm{m}}, \mathbf{r}^{\prime},-\omega_{0}\right) \mathrm{e}^{-\mathrm{i} \mathbf{k}_{0} \cdot \mathbf{r}^{\prime}} \mathrm{d} \mathbf{r}^{\prime} E_{0, \beta}\right|$.

In Eq. (3), the three phase factors $\phi_{\alpha}\left(\mathbf{r}_{\mathrm{m}}\right)$ (with $\alpha=x, y$ or $z$ ) are obtained by taking separately the phase of the three components of the complex number $\mathbf{E}\left(\mathbf{r}_{\mathrm{m}}, \omega\right)$. This splitting of both the phase factor and the Rabi frequency into three distinct components originates from the presence of evanescent fields [22,23].

At this stage, we have all the ingredients required to write Liouville's equation that governs the matrix density evolution of the molecule

$\dot{\rho}(t)=\frac{1}{\mathrm{i} \hbar}\left[H_{0}+W(t), \rho(t)\right]+\left[\mathscr{R}_{\text {spont }}+\mathscr{R}_{\mathrm{vib}}\right] \rho(t)$,

where the two Redfield operators $\mathscr{R}_{\text {spont }}$ and $\mathscr{R}_{\text {vib }}$, describe, respectively, the couplings with the photons bath and the internal vibrational states of the molecule [12]. This leads to a coupled system of four optical Bloch equations [12]:

$$
\begin{aligned}
& \dot{\rho}_{33}(t)=K \rho_{22}(t)-\Gamma_{3} \rho_{33}(t), \\
& \dot{\rho}_{22}(t)=-\left(K+\Gamma_{2}\right) \rho_{22}(t)-\mathrm{i} \Omega^{-} \rho_{12}(t)+\mathrm{i} \Omega^{+} \rho_{21}(t), \\
& \dot{\rho}_{12}(t)=\left[\mathrm{i} \delta_{L}-\frac{\Gamma_{2}}{2}\right] \rho_{12}(t)-\mathrm{i} \Omega^{+}\left(2 \rho_{22}(t)+\rho_{33}(t)-1\right), \\
& \dot{\rho}_{21}(t)=-\left[\mathrm{i} \delta_{L}+\frac{\Gamma_{2}}{2}\right] \rho_{21}(t)+\mathrm{i} \Omega^{-}\left(2 \rho_{22}(t)+\rho_{33}(t)-1\right),
\end{aligned}
$$

with

$\Omega^{-/+}=\sum_{\alpha=x, y, z} \frac{\Omega_{\alpha}\left(\mathbf{r}_{\mathrm{m}}\right)}{2} \mathrm{e}^{(-/+) \mathrm{i} \phi_{\alpha}\left(\mathbf{r}_{\mathrm{m}}\right)}$.

When the phase factors $\phi_{\alpha}\left(\mathbf{r}_{\mathrm{m}}\right)$ weakly vary from one field component $\alpha$ to another, we can put $\phi_{\alpha}\left(\mathbf{r}_{\mathrm{m}}\right) \equiv \phi\left(\mathbf{r}_{\mathrm{m}}\right)$ for 
$\alpha=x, y$ or $z$. In this case, the two factors $\Omega^{+}$and $\Omega^{-}$can be related to the usual Rabi frequency $\Omega\left(\mathbf{r}_{\mathrm{m}}\right)$ :

$\Omega^{+}=\left(\Omega^{-}\right)^{*}=\frac{\Omega\left(\mathbf{r}_{\mathrm{m}}\right)}{2} \mathrm{e}^{\mathrm{i} \phi\left(\mathbf{r}_{\mathrm{m}}\right)}$.

In Eqs. (7)-(10), we have also introduced four useful parameters, namely, (i) the detuning $\delta_{\mathrm{L}}=\omega_{2}-\omega_{0}$ between the laser and the resonant absorption frequency of the molecule, (ii) the vibrational relaxation constant $K$ between the excited state and the first excited singlet, and finally (iii) the two radiative decay rates of the molecule [15]:

$\Gamma_{2 / 3}=\Gamma_{2 / 3}^{(0)}+\frac{2 \mu_{2 / 3}^{2}}{\hbar} \operatorname{Im}\left\{\mathbf{S}\left(\mathbf{r}_{\mathrm{m}}, \mathbf{r}_{\mathrm{m}}, \omega_{2 / 3}\right)\right\}: \mathbf{u u}$,

where $\mathbf{u}$ is a unit vector that characterizes the molecule orientation, $\mu_{2 / 3}$ represent the transition dipole between the ground state and the $|2\rangle /|3\rangle$ levels, and $\Gamma_{2 / 3}^{(0)}$ are the spontaneous decay rates of the isolated molecule (Fig. 1). These parameters appear in the optical Bloch equations after application of the Redfield operator on the matrix density operator. In the present case, symmetry considerations imply that several Redfield operator matrix elements vanish [12]. The stationary population $\rho_{33}$ of the first singlet state $|3\rangle$ gives direct access to the detected fluorescence signal when the molecule is illuminated in permanent regime. In this case, the four terms on the left-hand side of Bloch equations tend simultaneously to zero. This leads to

$\rho_{33}=\left[1+\left(\frac{2}{K}+A_{1}+A_{2}\right) \Gamma_{3}\right]^{-1}$,

with

$A_{1}=\frac{\delta_{L}^{2}}{\Omega^{+} \Omega^{-}}\left\{\frac{1}{K}+\frac{1}{\Gamma_{2}}\right\}$

and

$A_{2}=\frac{\Gamma_{2}}{4 \Omega^{+} \Omega^{-}}\left\{1+\frac{\Gamma_{2}}{K}\right\}$.

The fluorescence signals generated by the molecule is then given by

$I_{\text {fluo }}\left(\mathbf{r}_{\mathrm{m}}\right)=\frac{\hbar \omega_{3} \Gamma_{3}}{1+\left(\frac{2}{K}+A_{1}+A_{2}\right) \Gamma_{3}}$.

Eq. (17) together with Eqs. (15) and (16) demonstrate the possibility of self-consistently bridging the gap between molecular and macroscopic scales. The entire optical information related to the dielectric environment is implicitly contained, via the field-susceptibility $\mathbf{S}$, in the factors $\Gamma_{2 / 3}$ and $\Omega^{+/-}$. Consequently, this approach is well-suited for investigating a large class of photophysical phenomena in confined geometries.

\section{Limiting cases for different illumination modes}

Another important advantage of this approach should also be emphasized. As illustrated by the previous analysis, a proper selection of the only active molecular levels for a given photophysical process leads to quasi-analytical expressions for the fluorescence signals. For example, two limiting cases particularly pertinent to on-going single molecule near-field optical experiments can be extracted from Eq. (17).

\subsection{Saturated absorption regime}

When the laser frequency $\omega_{0}$ excites the molecule at the absorption frequency $\omega_{2}$, the detuning factor $\delta_{L}$ in Eq. (15) and the factor $A_{1}$ tend towards zero. The magnitude of the excitation power can then be adjusted to approach the so-called saturation regime that occurs when the product $A_{2} \Gamma_{3}$ is much smaller than unity in Eq. (17). This condition is fulfilled when the Rabi frequency $\Omega\left(\mathbf{r}_{\mathrm{m}}\right)$ verifies the following relation (see Eqs. (12) and (16)):

$\Omega\left(\mathbf{r}_{\mathrm{m}}\right) \gg \sqrt{\Gamma_{2} \Gamma_{3}}$.

Moreover, for customary fluorescent molecules, the term $2 \Gamma_{3} / K$ in Eq. (17) remains always much smaller than unity. Consequently, within this asymptotic case, the fluorescence signal becomes merely proportional to the radiative decay $\Gamma_{3}$ :

$I_{\mathrm{sat}}\left(\mathbf{r}_{\mathrm{m}}\right) \simeq \hbar \omega_{3} \Gamma_{3}\left(\mathbf{r}_{\mathrm{m}}, \omega_{3}\right)$.

After substitution of Eq. (13) into Eq. (19), the variation of $I_{\text {fluo }}\left(\mathbf{r}_{\mathrm{m}}\right)$ with respect to the molecule location $\mathbf{r}_{\mathrm{m}}$ is found to be proportional to the imaginary part of the field susceptibility $\mathbf{S}$ associated with the entire environment,

$I_{\text {sat }}\left(\mathbf{r}_{\mathrm{m}}\right) \simeq \hbar \omega_{3} \Gamma_{3}^{(0)}+2 \mu_{3}^{2} \omega_{3} \operatorname{Im}\left\{\mathbf{S}\left(\mathbf{r}_{\mathrm{m}}, \mathbf{r}_{\mathrm{m}}, \omega_{3}\right)\right\}: \mathbf{u u}$.

For a specific orientation of the molecule along the $\alpha$-direction, the factor $\operatorname{Im}\left\{\mathbf{S}\left(\mathbf{r}_{\mathrm{m}}, \mathbf{r}_{\mathrm{m}}, \omega_{2 / 3}\right)\right\}$ :uu can be related to the partial photonic LDOS $n_{\alpha}\left(\mathbf{r}_{\mathrm{m}}, \omega_{3}\right)$ at the fluorescence frequency $\omega_{3}$ [14],

$n_{\alpha}\left(\mathbf{r}_{\mathrm{m}}, \omega_{3}\right)=\frac{1}{2 \pi^{2} \omega_{3}} \operatorname{Im}\left\{\mathbf{S}_{\alpha, \alpha}\left(\mathbf{r}_{\mathrm{m}}, \mathbf{r}_{\mathrm{m}}, \omega_{3}\right)\right\}$.

Finally, substitution of Eq. (21) into Eq. (20) leads to

$I_{\text {sat }}\left(\mathbf{r}_{\mathrm{m}}\right)=\hbar \omega_{3} \Gamma_{3}^{(0)}+4 \omega_{3}^{2} \mu_{3}^{2} \pi^{2} n_{\alpha}\left(\mathbf{r}_{\mathrm{m}}, \omega_{3}\right)$.

Consequently, when the saturation regime is reached, the molecule tends to gradually forget any initial information related to the structure of the excitation field. In this case, the molecule loses the history of the successive absorption events and emits fluorescence photons that probe the partial photonic LDOS associated with the environment. Such experimental conditions can be obtained by working at low temperature in order to 
minimize the absorption linewidth $\Gamma_{2}$ of the molecular probe. Very recently, Michaelis et al. succeeded in operating a low temperature SNOM configuration where the source of light was reduced to a single fluorescing terrylene molecule embedded in a paraterphenyl microcrystal, which itself was glued on the apex of a sharpened optical fiber [7,24]. At $1.4 \mathrm{~K}$, the terrylene linewidth is about $47 \mathrm{MHz}$, which provides ideal conditions to observe, under saturated conditions, the variations of the LDOS as the micro-crystal is raster-scanned [25].

A simulation of this operation mode is presented in Fig. 2b, where a single terrylene molecule raster-scans the sample depicted in Fig. 2a. The fluorescence wavelength $\lambda_{3}=2 \pi c / \omega_{3}$ of terrylene is $630 \mathrm{~nm}$ and its fluorescence transition dipole $\mu_{3}$ oriented along the $y$-axis. According to Eq. (22), the molecule signal is modulated by the partial $y$-LDOS computed at the fluorescence wavelength $\lambda_{3}$. The presence of symmetrical ripples around the dielectric cylinders indicates that a LDOS map characterizes a given system independently of the illumination mode [26]. Let us note that the other partial $x$-LDOS can be probed by rotating the molecule by $\pi / 2$ in the $x y$-plane.

\subsection{Off-saturation absorption regime [27]}

In this second asymptotic case, we also maintain the detuning factor $\delta_{\mathrm{L}}$ close to zero and consider the fluores- cence signal when the molecule is excited at a low Rabi frequency. This regime is described by the following inequality,

$\Omega\left(\mathbf{r}_{\mathrm{m}}\right) \ll \sqrt{\Gamma_{2} \Gamma_{3}}$,

which corresponds to the condition $A_{2} \Gamma_{3} \gg 1$. The fluorescence signals $I_{\text {off-sat }}\left(\mathbf{r}_{\mathrm{m}}\right)$ reads then

$I_{\text {off-sat }}\left(\mathbf{r}_{\mathrm{m}}\right) \simeq \frac{\hbar \omega_{3}}{A_{2}}=\frac{4 \hbar \omega_{3} K \Omega_{+} \Omega_{-}}{\Gamma_{2}\left(K+\Gamma_{2}\right)}$.

In the case of low near-field phase variations (Eq. (12)), this signal becomes proportional to the square of the Rabi frequency $\Omega\left(\mathbf{r}_{\mathrm{m}}\right)$ at the molecule location:

$I_{\text {off-sat }}\left(\mathbf{r}_{\mathrm{m}}\right)=A \Omega^{2}\left(\mathbf{r}_{\mathrm{m}}\right)$,

with $A=\hbar \omega_{3} K / \Gamma_{2}\left(K+\Gamma_{2}\right)$. For a particular orientation of the molecule along the $\alpha$-direction, $\Omega^{2}\left(\mathbf{r}_{\mathrm{m}}\right)=$ $\mu_{2}^{2} \mathbf{E}_{\alpha}^{2}\left(\mathbf{r}_{\mathrm{m}}\right)$, and the molecule probes the local near-field intensity $[27,28]$,

$I_{\text {off-sat }}\left(\mathbf{r}_{\mathrm{m}}\right)=A \mu_{2}^{2} \mathbf{E}_{\alpha}^{2}\left(\mathbf{r}_{\mathrm{m}}\right)$.

This result shows that, unlike what happened in the fluorescence saturation regime where the molecule probed the photonic LDOS (Eq. (22)), the off-saturation regime leads to a fluorescence signal that is proportional to the local near-field intensity. According to Eq. (23), this condition is easily verified when the Rabi frequency is small compared to the absorption linewidth. These

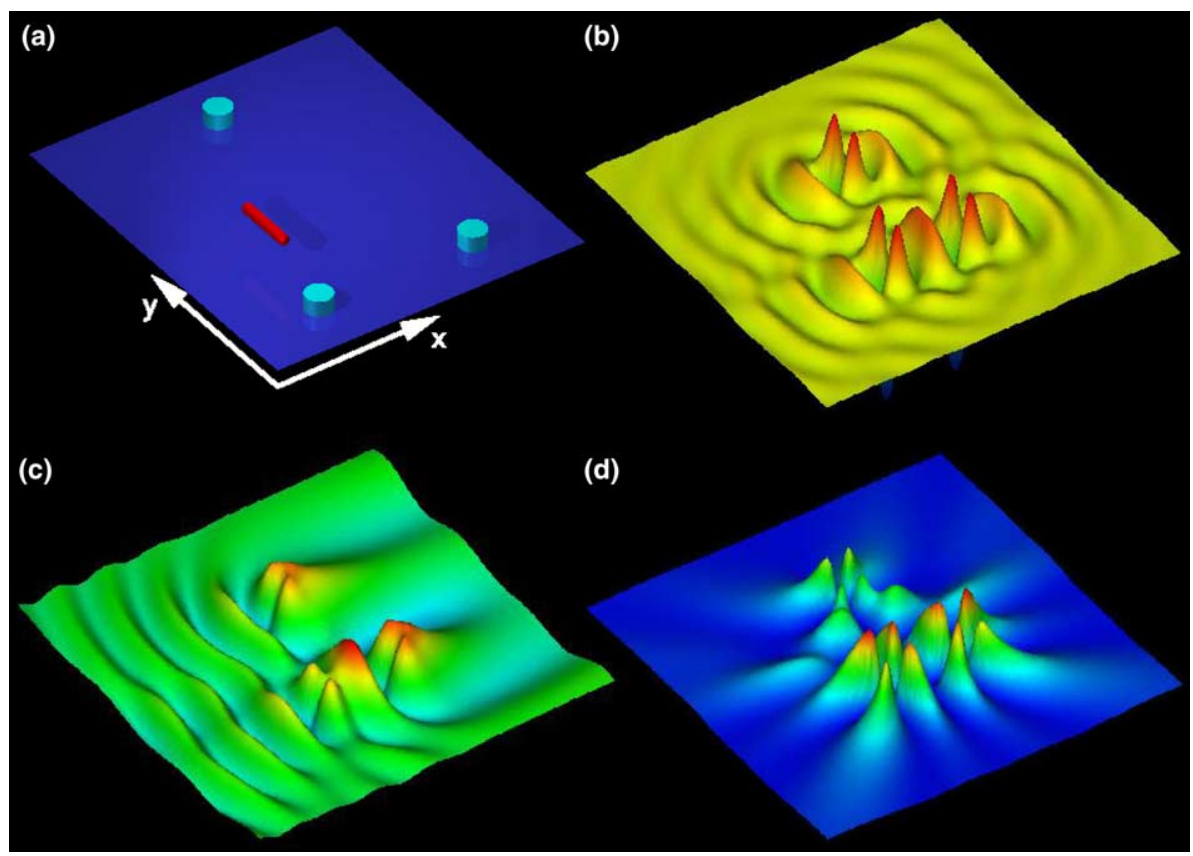

Fig. 2. (a) Geometry used for the calculation: three dielectric cylindrical protrusions (diameter $100 \mathrm{~nm}$, height $60 \mathrm{~nm}, \varepsilon=4.41$ ) are placed at the corners of an isosceles triangle with base $600 \mathrm{~nm}$ and perpendicular $1000 \mathrm{~nm}$ on a glass surface $(\varepsilon=2.25)$. The red ellipsoid represents the molecule that raster-scans the sample at a constant height $z=150 \mathrm{~nm}$ above the surface. The system is illuminated from underneath in total internal reflection with s-polarization. (b) Fluorescence signal computed in the saturation regime (Eq. (22)) when the molecule raster-scans the sample. (c) and (d) Fluorescence signal computed in the off-saturation regime (Eq. (26)), for two different propagation directions of the illumination field: $y$-direction (c) and $x$-direction (d). Map dimensions: (a) $1.2 \times 1.4 \mu \mathrm{m}^{2}$, (b)-(d) $3 \times 3 \mu \mathrm{m}^{2}$. 
conditions have been used in a series of beautiful experiments performed at room temperature where the detection of local signals emitted by individual molecules was reported $[2,28]$. In particular, it was demonstrated in Ref. [2] how individual fluorescing molecules were able to precisely map the electric field intensity generated at the apex of a metal coated tip, thereby providing the first direct verification of Bethe's theory of light diffraction by a subwavelength aperture.

Two simulations of this off-saturation mode, calculated at the illumination wavelength $\lambda_{2}=578 \mathrm{~nm}$, are given in Figs. $2 c$ and d. In this case, it may be seen that the molecule probes the intensity $\mathbf{E}_{y}^{2}$ associated with the $y$-component of the optical electric near-field confined by the environment. Unlike what happened in the saturated absorption regime (Fig. 2b), the recorded fluorescence signal dramatically depends on the illumination conditions and does not provide direct information on the sole topography of the sample.

\subsection{Arbitrary illumination regime}

When neither conditions examined under (i) or (ii) apply, no evident simplification can be made and the detected fluorescence signal must be computed from the general relation (17). In this case, information related to the electric field distribution is entangled with local information related to the LDOS calculated at the fluorescence frequency.

\section{Summary}

In conclusion, we have developed a unified formalism to describe molecular photophysical processes in a confined geometry. This approach includes a non-perturbative quantum treatment of all the different active molecular levels, as well as the complex and rapidly varying field distributions associated with a complicated optical environment. The combination of these two features renders the method particularly powerful for the accurate analysis of experiments performed with local probe techniques and single active molecules. The obtained equations provide new insights into the signal effectively radiated in the near-field by a single molecule. Important limiting cases in the saturated and offsaturated absorption regimes can be recovered from this general formalism.

\section{Acknowledgements}

O.J.F.M. gratefully acknowledges support from the Swiss National Science Foundation. This work was supported in part by the European Network of Excellence (NoE) Plasmo-Nano-Devices (Contract No. 507879).

\section{References}

[1] K. Lieberman, S. Harush, A. Lewis, R. Kopelman, Science 247 (1990) 59.

[2] E. Betzig, R.J. Chichester, Science 262 (1993) 1422.

[3] W.E. Moerner, T. Plakhotnik, T. Irngartinger, U.P. Wild, D.W. Pohl, B. Hecht, Phys. Rev. Lett. 73 (1994) 2764.

[4] E. Betzig, J.K. Trautman, J.D. Harris, J.S. Weiner, R.L. Kostelack, Science 251 (1991) 1468.

[5] W.L. Barnes, J. Modern Opt. 45 (1998) 661.

[6] W.E. Moerner, M. Orrit, Science 283 (1999) 1671.

[7] J. Michaelis, J.M.C. Hettich, V. Sandogdhar, Nature 405 (2000) 325.

[8] P. Andrew, W.L. Barnes, Science 290 (2000) 785.

[9] J.A. Nieminen, S. Paavilainen, Phys. Rev. B 60 (1999) 2921.

[10] O.J.F. Martin, C. Girard, A. Dereux, Phys. Rev. Lett. 74 (1995) 526.

[11] P. de Vries, D.V. van Coeverden, A. Lagendijk, Rev. Mod. Phys. 70 (1998) 447.

[12] C. Cohen-Tannoudji, J. Dupont-Roc, G. Grynberg, Processus D'interaction Entre Photons Et Atomes, InterEditions, Paris, 1988.

[13] C.M. Bowden, J.P. Dowling, Phys. Rev. A 47 (1993) 1247.

[14] G. Colas des Francs, C. Girard, J.C. Weeber, C. Chicanne, T. David, A. Dereux, D. Peyrade, Phys. Rev. Lett. 86 (2001) 4950.

[15] G.S. Agarwal, Phys. Rev. A 12 (1975) 1475.

[16] P. Das, H. Metiu, J. Phys. Chem. 89 (1982) 4680.

[17] N. Vats, S. John, K. Busch, Phys. Rev. A 65 (2002) 43808.

[18] C. Girard, O.J.F. Martin, A. Dereux, Phys. Rev. Lett. 75 (1995) 3098.

[19] L. Novotny, Appl. Phys. Lett. 69 (1996) 3806.

[20] C. Henkel, V. Sandoghdar, Opt. Commun. 158 (1998) 250.

[21] G. Parent, D. VanLabeke, D. Barchiesi, J. Microscopy 194 (1999) 281.

[22] R. Carminati, Phys. Rev. E 55 (1997) R4901.

[23] M.L.M. Balistreri, J.P. Korkerik, L. Kuipers, N.F. Van Hulst, Phys. Rev. Lett. 85 (2000) 294.

[24] V. Sandogdhar, Phys. World 14 (2001) 29.

[25] G. Colas des Francs, C. Girard, A. Dereux, J. Chem. Phys. 117 (2002) 4659.

[26] G. Lévêque, G. Colas des Francs, C. Girard, J.C. Weeber, C. Meier, C. Robillard, R. Mathevet, J. Weiner, Phys. Rev. E. 65 (2002) 36701.

[27] T. Plakhotnik, E. Donley, U.P. Wild, Ann. Rev. Phys. Chem. 48 (1997) 181.

[28] B. Sick, B. Hecht, L. Novotny, Phys. Rev. Lett. 85 (2000) 4482. 